SLAC-PUB-6589

TAUP-2240/95

CERN-TH/95-68

hep-ph/9503411

\title{
Comparison of the Padé Approximation Method to Perturbative QCD Calculations ${ }^{\star \dagger}$
}

\author{
Mark A. SAmuel \\ Department of Physics \\ Oklahoma State University \\ Stillwater, OK 74078 \\ e-mail: physmas@mvs.ucc.okstate.edu \\ and \\ Stanford Linear Accelerator Center \\ Stanford University, Stanford, California 94309 \\ and \\ JOHN ELLIS \\ CERN \\ Geneva \\ Switzerland \\ e-mail: johne@cernvm.cern.ch \\ and \\ MAREK KARLINER \\ School of Physics and Astronomy \\ Tel-Aviv University \\ Tel-Aviv, Israel \\ e-mail:marek@vm.tau.ac.il
}

\begin{abstract}
We present a method of estimating perturbative coefficients in Quantum Field Theory using Padé Approximants. We test this method on various known QCD results, and find that the method works very well.
\end{abstract}

$\star$ Work supported by the Department of Energy, contract DE-AC03-76SF00515.

$\dagger$ To appear in Phys. Rev. Lett. 
By using the first $n$ coefficients in a series expansion, we have estimated the $(n+1)$-st perturbative coefficient in Quantum Field Theory (QFT). Though there is currently no theoretical basis for extrapolating coefficients in the perturbative loop expansion of QFT by our method, our results have thus far been in good agreement with the calculated coefficients of quantum electrodynamics (QED), as well as with series in statistical physics, condensed matter theory and mathematics [1-6]. In this paper we compare our method to the perturbative loop expansion of quantum chromodynamics (QCD) at the five-loop level. We shall present results for the $R$-ratio, the $R_{\tau}$ ratio, the QCD $\beta$ Function and two QCD Sum Rules.

Our method makes use of Padé Approximants (PA) and enables us to obtain an estimate and an error-bar for each coefficient. We define the PA

$$
[N / M]=\frac{a_{0}+a_{1} X+\cdots+a_{N} X^{N}}{1+b_{1} X+\cdots+b_{M} X^{M}}
$$

to the series $S$ where we set

$$
[N / M]=S+\mathcal{O}\left(X^{N+M+1}\right) \quad \text { and } \quad S=\sum_{n=0}^{\infty} S_{n} X^{n} .
$$

One solves Eq. (2) and then predicts the coefficient of the next term $S_{N+M+1}$. This is what we do in this paper. One can also use the full PA to estimate the sum of the whole series $S$. This is what we will do in the future $[7,8]$. For a detailed description see Refs. [5] and [6].

The PA's are known to accelerate the convergence of many series by including the effects of higher (unknown) terms, thus providing a more accurate estimate of the series. We have recently proved the following theorem, which provides a 
useful sufficient condition for the PA's to be accurate. Defining $f(n) \equiv \ell n S_{n}$ and considering

$$
g(n)=\frac{d^{2} f(n)}{d n^{2}}
$$

a sufficient condition for the PA's to converge is that

$$
\lim _{n \rightarrow \infty} g(n)=0
$$

The PA's thus provide reliable estimates of asymptotic series whose coefficients diverge as

$$
S_{n}=n ! k^{n} n^{\gamma}
$$

as is believed to be the case in QED and QCD [9]. It can easily be shown that Eq. (4) is satisfied for $S_{n}$ given by Eq. (5). In the cases of these and other series whose Borel transform has a finite radius of convergence, the higher-order PA's give progressively better approximations to the Principal Value of the transform integral over Borel singularities. It is easy to check that for series with one or two simple Borel poles, (i.e. IR and UV renormalons), Padé approximants predict the next term in a given series with a rapidly increasing precision. In these cases an analytic estimate $\sim M ! / N^{M}$ can be made for the relative error of the $[N / M]$ Padé approximant prediction of the next term in the series.

It might be objected, however, that these cases are not sufficiently complicated to be realistic. Therefore, as an exercise, we have evaluated Padé approximants to the large- $N_{f}$ limit of the vacuum polarization D-function in QCD, which is known to all orders in $\alpha_{s}$, and whose Borel transform contains an infinite series of 
double poles at both positive and negative integers. ${ }^{10}$ Once again, as seen in the Figure, the Padé approximants' predictions of the next term in the series converge rapidly, in agreement with the above-mentioned estimated error for the $[N / M]$ Padé approximant. The convergence of the Borel transform of the D-function series, in particular, indicates that our PA approach is well suited for perturbation series with the asymptotic behavior expected in QCD.

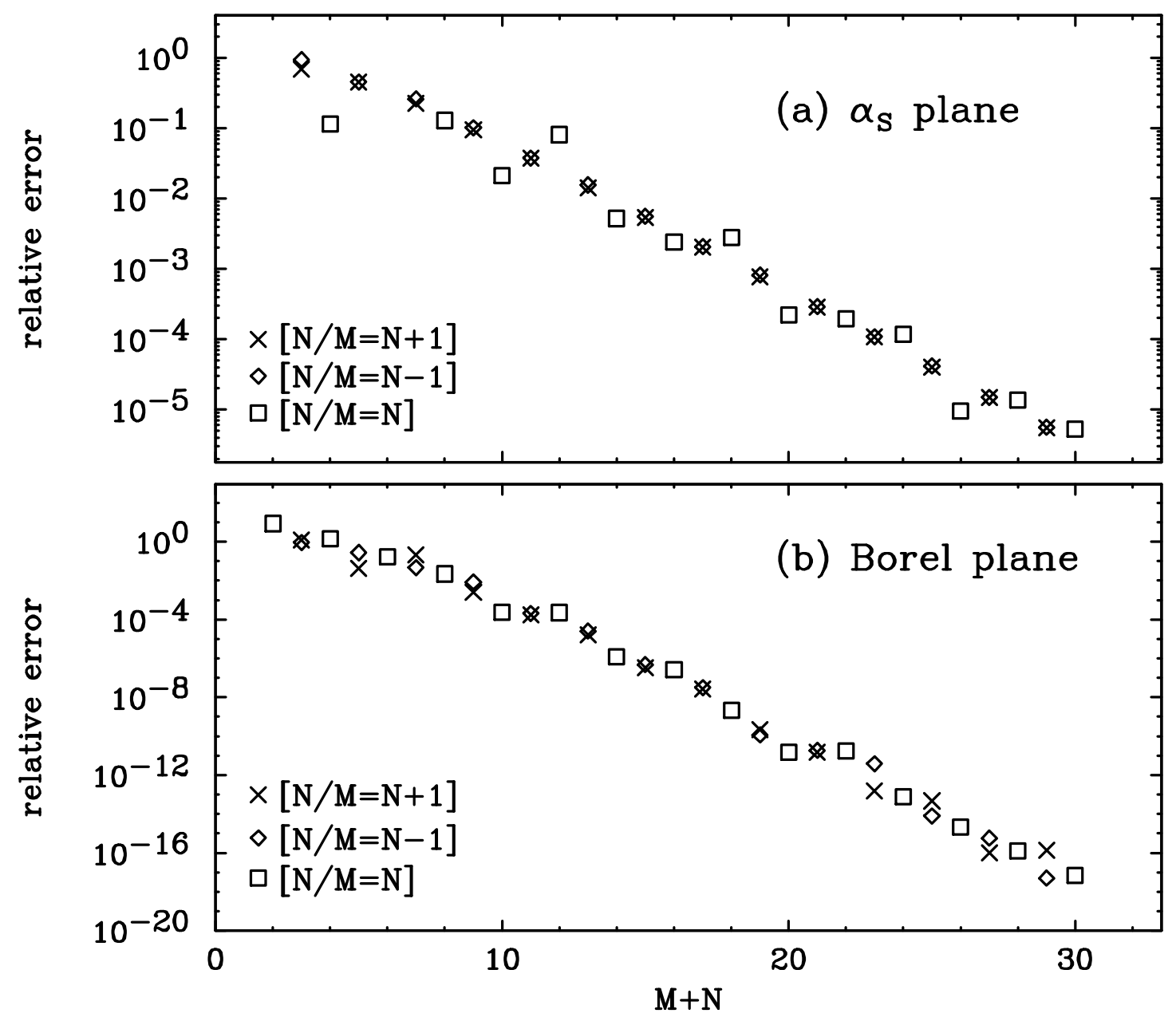

Relative errors in the $[N / M]$ Padé approximants: (a) to the QCD vacuum polarization D-function, evaluated to all orders in the large- $N_{f}$ approximation ${ }^{10}$ - the rate of convergence agrees with expectations for a series with a discrete set of Borel poles, and (b) to the Borel transform of the D-function series, where the convergence is particularly striking. 
We now turn to some QCD applications of our PA approach. Let us first consider the $R_{\tau}$ ratio ${ }^{11}$ where $R_{\tau}$ is defined as follows:

$$
R_{\tau} \equiv \Gamma(\tau \rightarrow \nu+\text { hadrons }) / \Gamma(\tau \rightarrow e \nu \bar{\nu})=3 S_{\mathrm{EW}}\left(r_{\tau}+r_{\tau}^{1}\right)
$$

where $S_{\mathrm{EW}}=1.019$ is the electroweak correction and $r_{\tau}^{1}=-1.58 \%$ is the nonperturbative contribution. The perturbative QCD contribution is given by

$$
r_{\tau}=1+\frac{\alpha_{s}}{\pi}+5.202\left(\frac{\alpha_{s}}{\pi}\right)^{2}+26.36\left(\frac{\alpha_{s}}{\pi}\right)^{3}+(109.2 \pm 12.9)\left(\frac{\alpha_{s}}{\pi}\right)^{4}
$$

where the last coefficient is our estimate of the five-loop contribution. Using the experimental average ${ }^{12,13}$ value $R_{\tau}=3.623(17)$, we obtain for the strong coupling constant

$$
\alpha_{s}\left(M_{\tau}\right)=0.325(6)
$$

where the error does not include various systematic uncertainties, which go beyond the scope of this letter and are discussed elsewhere. ${ }^{8}$ Numerically Eq. (7) becomes $r_{\tau}=1+0.1035+0.0557+0.0292+0.0125=1.201(19)$. One can see that the perturbative series is converging, albeit somewhat slowly. Using the known $\beta$ function (see Table II for our estimate of the four-loop $\beta$ function), Eq. (8) corresponds to $\Lambda^{(3)}=355(11) \mathrm{MeV}$ in the $\overline{\mathrm{MS}}$ Scheme. Stepping up through the 4 and 5 fermion-thresholds $\left(m_{c}=1.5 \mathrm{GeV}\right.$ and $m_{b}=5 \mathrm{GeV}$, respectively) we get $\Lambda^{(4)}=306(11) \mathrm{MeV}$ and $\Lambda^{(5)}=218(9) \mathrm{MeV}$, and, hence,

$$
\alpha_{s}(34 \mathrm{GeV})=0.1399(11)
$$


For the $R$ ratio, we have ${ }^{14}$

$R=3 \Sigma Q_{f}^{2} r \quad: \quad r=1+\left(\frac{\alpha_{s}}{\pi}\right)+1.409\left(\frac{\alpha_{s}}{\pi}\right)^{2}-12.805\left(\frac{\alpha_{s}}{\pi}\right)^{3}-(87.5 \pm 10.8)\left(\frac{\alpha_{s}}{\pi}\right)^{4}$

The last term in Eq. (10) is our estimate for the five-loop contribution to $R$ where we here extrapolated the related Adler D-function. Using Eq. (9) we obtain

$$
r(34 \mathrm{GeV})=1+0.0445+0.0028-0.0011-0.0003=1.0459(4) .
$$

This series for $r$ in Eq. (11), where the contributions in each order, up to five loops, are given, seems to converge nicely. Experimentally there are two measurements of $r(34 \mathrm{GeV})$. They are $r(34 \mathrm{GeV})=1.049(7)^{15}$ and $r(34 \mathrm{GeV})=1.056(8)^{16}$. It can be seen that the extrapolation prediction in Eq. (11) is in good agreement with these experimental values. We can now evolve $\alpha_{s}$ up to $M_{Z}$, the $Z$ boson mass. Our result is

$$
\alpha_{s}\left(M_{Z}\right)=0.119(2)
$$

which is consistent with the latest experimental value obtained from total crosssection measurements at $\mathrm{LEP}^{17}$

$$
\alpha_{s}\left(M_{Z}\right)=0.126(6)
$$

For $r$ at $M_{Z}$ we get $r\left(M_{Z}\right)=1+0.0378+0.0020-0.0007-0.0002=1.0389(2)$.

We now present our estimates for higher-order perturbative coefficients for $R$, $R_{\tau}$, the QCD $\beta$ Function and two QCD Sum Rules. All of the results presented here are in the $\overline{\mathrm{MS}}$ scheme. The corresponding results for other schemes can also 
be obtained. However the scheme-dependence should decrease in higher order, disappearing if all orders are known.

To provide systematic-error estimates, we first apply our method to $S_{n}$, obtaining our estimate $S_{n+1}^{(0)}$. We then apply it to the reciprocals $r_{n} \equiv 1 / S_{n}$, and take the reciprocal again to obtain $S_{n+1}^{(1)}$. We then take differences $t_{n}=r_{n+1}-r_{n}$ and apply our method to obtain a third estimate $S_{n+1}^{(2)}$. Our systematic-error estimate is $\Delta / 2$, where $\Delta=\left|S_{n+1}^{(2)}-S_{n+1}^{(1)}\right|$. We then combine the diagonal and non-diagonal estimates of $S_{n+1}^{(0)}$, weighted by $1 / \Delta^{2}$, to obtain our final estimate for $S_{n+1}$.

In Table I we present our results for $R$ and $R_{\tau}$. The first entry in each case is the four-loop result. The light-by-light contribution is small but should be added to $R(\overline{\mathrm{MS}})$. Our estimates of the four-loop coefficients, based on PA's to lower-order coefficients, agree well with the known exact results, providing a sound phenomenological footing for our method. The second entries are our estimates for the five-loop coefficients and the numbers in brackets are our estimate of the systematic errors. The results $-96.8 \mathrm{KS}$ and 105.5 KS are the estimates of Ref. [18], obtained using a completely independent method, Optimized Perturbation Theory (OPT). The agreement with our estimates is very good.

In Table II we present our results for the QCD $\beta$-function. The agreement with the known 3-loop results is very good, and we present the first estimates of the four-loop QCD $\beta$ function. Note that the three- and four-loop results are schemedependent, and we use the $\overline{M S}$ scheme throughout. The 3-loop result is the same for any MS-type scheme. Since we use the $\overline{M S}$ result for the 3-loop coefficient our estimate for the four-loop coefficient is also for the $\overline{M S}$ scheme. 
In Table III we present our results for the Bjorken sum rule for deep inelastic (unpolarized) neutrino-nucleon scattering. To compare with Ref. [18], we multiply their results BjnSR by $-2 / 3$. Our results for $\mathcal{O}\left(\alpha_{s}^{3}\right)$ are very good again, and again the agreement with Ref. [18] in $\mathcal{O}\left(\alpha_{s}^{4}\right)$ is excellent. We present our results for the Bjorken sum rule for deep inelastic polarized electron-nucleon scattering, -BjpSR in Table IV. Again our results in $\mathcal{O}\left(\alpha_{s}^{3}\right)$ are good and our $\mathcal{O}\left(\alpha_{s}^{4}\right)$ estimates agree with Ref. [18]. The Gross-Llewellyn Smith sum rule (GLSSR) differs from the BjpSR by the light-by-light contribution: $G L S S R=B j p S R-0.413 f$. The lightby-light contribution here and for $R$ should be treated separately. This contribution is small, however, for all cases of interest.

In Table $\mathrm{V}$ we present our results for the $R$ ratio for various $N_{f}$. The small difference for $N_{f}=5$ compared with Table I is due to a slight difference in averaging the various estimates. The results for the four-loop coefficients are excellent and the agreement with Ref. [18] for the five-loop coefficients is satisfying.

Although it is interesting that our estimates for the next term agree with Ref. [18] and we believe both of us are right, we cannot be certain. Both of us may be wrong. Fortunately, even a crude estimate of the next term is sufficient since their contributions to the full series are small. However, it is important to know they are small!

In conclusion, we have used our estimation method, which makes use of Padé Approximants, to estimate various perturbative coefficients in QCD. Our estimates for the known terms is very good. Moreover our estimates for the next unknown terms agree very well with the results of Ref. [18] in all cases where comparison is possible. 
Note added:

A phenomenological extraction of the five-loop D-function coefficient from measured moments of $\tau$ decay data has recently appeared ${ }^{19}$, which confirms our prediction.

In the process of our analysis we are comparing our results with explicit expressions given in ref. 14 and in papers listed in ref. 18. The original references for the NNLO calculations are: $R(s)$ - Ref. 14 and S.G.Gorishny, A.L.Kataev, S.A.Larin in "Standard Model and Beyond: from LEP to UNK and LHC" Dubna, USSR, October 1-5,1990, S. Dubnicka, et al., Eds. World Scientific (1991) p.288; the QCD beta-function - O.V. Tarasov, A.A. Vladimirov and A.Yu. Zharkov, Phys. Lett. B93(1980)429; the Bjorken non-polarized sum rule - S.A. Larin, F.V. Tkachov and J. Vermaseren, Phys. Rev. Lett. 66(1991)862 and Bjorken polarized sum rule - S.A.Larin and J. Vermaseren, Phys. Lett. B259(1991)345. We thank Andrei Kataev for correspondence on this matter.

Acknowledgements:

We would like to thank the theory group at SLAC for its kind hospitality. We would also like to thank the following people for very helpful discussions: David Atwood, Richard Blankenbecler, Stan Brodsky, N. Deshpande, JoAnne Hewett, Helen Perk, Jacques Perk, Dominique Pouliot, Helen Quinn, Tom Rizzo, Davison Soper, Levan Surguladze and N.V.V.J. Swamy. This work was supported by the U.S. Department of Energy under grant numbers DE-FG05-84ER40215 and DE-AC0376SF00515. The research of M.K. was supported in part by grant No. 90-00342 from the United States-Israel Binational Science Foundation (BSF), Jerusalem, Is- 
rael, and by a Grant from the G.I.F., the German-Israeli Foundation for Scientific Research and Development.

\section{REFERENCES}

1. M. A. Samuel, G. Li and E. Steinfelds, Phys. Rev. D48, 869 (1993).

2. M. A. Samuel and G. Li, Int. J. Th. Phys. 33, 1461 (1994).

3. M. A. Samuel, G. Li and E. Steinfelds, Phys. Lett. B323, 188 (1994).

4. M. A. Samuel and G. Li, Phys. Lett. B331, 114 (1994).

5. M. A. Samuel, G. Li and E. Steinfelds, "On Estimating Perturbative Coefficients in QFT and Statistical Physics," Phys. Rev. E (to be published, 1995).

6. M. A. Samuel, "On Estimating Perturbative Coefficients in QFT, Statistical Physics and Mathematics," Oklahoma State University Research Note 290, July (1994).

7. J. Ellis, M. Karliner, M. Samuel and E. Steinfelds, "The Anomalous Magnetic Moments of the Electron and the Muon-Higher Precision with Padé Approximants," SLAC-PUB-6670 (1994), hep-ph/9409376.

8. J. Ellis, M. Karliner and M. Samuel, "Towards an Improved Determination of $\alpha_{s}$ Using Higher-Order Perturbative QCD." (in preparation).

9. A. I. Vainshtein and V. I. Zakharov, Phys. Rev. Lett. 73, 1207 (1994).

10. C.N. Lovett-Turner and C.J. Maxwell, Nucl. Phys. B432, 147 (1994). 
11. E. Braaten, Phys. Rev. Lett. 71, 1316 (1993) and references therein.

12. M. Davier, Proc. 2-nd Workshop on $\tau$ Lepton Physics, Ohio State U., Columbus, Ohio, Sep. 8-11, 1992, pg. 514, K. K. Gan, Ed., World Scientific (1993).

13. W. Trischuk, Proc. 2-nd Workshop on $\tau$ Lepton Physics, op. cit., pg. 59; W. J. Marciano, Proc. DPF-92 Meeting, Fermilab, Nov. (1992).

14. L. R. Surguladze and M. A. Samuel, in Beyond the Standard Model II, Norman, Oklahoma, Nov. 1-3, 1990; K. Milton, R. Kantowski and M. A. Samuel, Eds., World Scientific (1991); L. R. Surguladze and M. A. Samuel, Phys. Rev. Lett. 66, 560 (1991); S. G. Gorishny, A. L. Kataev and S. A. Larin, Phys. Lett. B259, 144 (1991).

15. J. J. Hernandez et al., Phys. Lett. B239, 1 (1990). W. De Boer, "Experimental Results on QCD from $e^{+} e^{-}$Annihilation," SLAC-PUB-4428 (1987), invited talk at the 10th Warsaw Symposium on Elementary Particle Physics, Kazimierz, Poland, May 25-29, 1987, p 503.

16. G. D'Agostini, W. De Boer and G. Grindhammer, Phys. Lett. B229, 160 (1989).

17. LEP Electroweak Working Group, CERN-PPE-94-187, Nov 1994, Contributed to ICHEP 94, Glasgow, 1994.

18. A. L. Kataev and V. V. Starshenko, Mod. Phys. Lett. A10, 235 (1995).

19. F. Le Diberder, Experimental estimates of higher order perturbative corrections to $R_{\tau}$, Orsay preprint, LAL-94-43, submitted to Phys. Lett. B. 


\section{TABLE I}

Estimates for $R(\overline{\mathrm{MS}}), N_{f}=5$, and $R_{\tau}(\overline{\mathrm{MS}}), N_{f}=3$, at the four- (first row) and five-loop (second row) order. The numbers in brackets are the estimated $1 \sigma$ error-bars. The four-loop results are compared with the exact (known) results and the five-loop results are compared with those of KS. $N_{f}$ is the number of fermions (quarks).

\begin{tabular}{lrr} 
SERIES & ESTIMATE & EXACT \\
\hline$R(\overline{\mathrm{MS}}), N_{f}=5$ & $-10.20(1.53)$ & -12.76 \\
& $-87.5(10.8)$ & $-96.8(\mathrm{KS})$ \\
$R_{\tau}(\overline{\mathrm{MS}}), N_{f}=3$ & $27.06(6.77)$ & 26.37 \\
& $109.2(12.9)$ & $105.5(\mathrm{KS})$ \\
\hline
\end{tabular}

\section{TABLE II}

Estimates for the QCD $\beta$-function in three- (first row) and four-loop (second row) order. The 3 -loop results are compared with the exact (known) results. $N_{f}$ is the number of fermions (quarks).

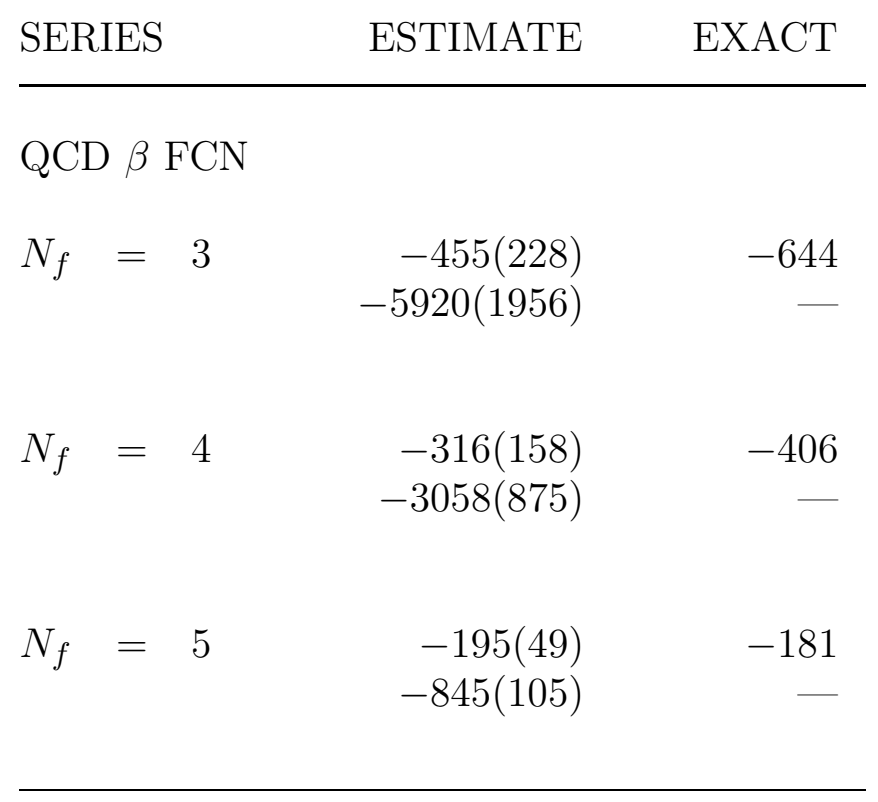




\section{TABLE III}

Estimates for the Bjorken sum rule for deep inelastic (unpolarized) neutrinonucleon scattering. The $\mathcal{O}\left(\alpha_{s}^{3}\right)$ (first row) results are compared with the exact (known) results and the $\mathcal{O}\left(\alpha_{s}^{4}\right)$ (second row) results are compared with those of KS. We multiply their results by $-2 / 3$ for ease of comparison.

\begin{tabular}{|c|c|c|c|c|}
\hline \multicolumn{3}{|c|}{ SERIES } & ESTIMATE & EXACT \\
\hline \multicolumn{5}{|c|}{ BjnSR $X-2 / 3$} \\
\hline$N_{f}$ & $=$ & 3 & $\begin{array}{r}-13.0(6.0) \\
-116(69)\end{array}$ & $\begin{array}{r}-18.6 \\
-133(\mathrm{KS})\end{array}$ \\
\hline$N_{f}$ & $=$ & 4 & $\begin{array}{r}-10.5(4.7) \\
-67.2(17.7)\end{array}$ & $\begin{array}{r}-13.4 \\
-75.8(\mathrm{KS})\end{array}$ \\
\hline$N_{f}$ & $=$ & 5 & $\begin{array}{r}-8.3(3.6) \\
-30.6(1.4)\end{array}$ & $\begin{array}{r}-8.5 \\
-29.4 \text { (KS) }\end{array}$ \\
\hline
\end{tabular}

TABLE IV

Estimates for the Bjorken sum rule for deep inelastic polarized electron-nucleon scattering. The $\mathcal{O}\left(\alpha_{s}^{3}\right)$ (first row) results are compared with the exact (known) results and the $\mathcal{O}\left(\alpha_{s}^{4}\right)$ (second row) results are compared with those of KS. Their result should be multiplied by -1 to compare with ours.

\begin{tabular}{lrr} 
SERIES & ESTIMATE & EXACT \\
\hline- BjpSR & & \\
$N_{f}=3$ & $-12.8(6.4)$ & -20.2 \\
& $-112(33)$ & $-130(\mathrm{KS})$ \\
$N_{f}=4$ & $-10.6(4.4)$ & -13.9 \\
& $-58.2(15.2)$ & $-68.1(\mathrm{KS})$ \\
$N_{f}=5$ & $-8.5(3.4)$ & -7.8 \\
& $-21.1(3.4)$ & $-17.8(\mathrm{KS})$ \\
\hline
\end{tabular}




\section{TABLE V}

Estimates for $R(\overline{\mathrm{MS}})$ for various $N_{f}$ at the four- (first row) and five-loop (second row) order. The four-loop results are compared with the exact (known) results and the five-loop results are compared with those of KS.

\begin{tabular}{lrr} 
SERIES & ESTIMATE & EXACT \\
\hline R RATIO & & \\
$N_{f}=3$ & $-14.1 \pm 2.0$ & -10.27 \\
& $-119.3 \pm 6.3$ & $-128.4(\mathrm{KS})$ \\
$N_{f}=4$ & $-12.2 \pm 1.4$ & -11.5 \\
& $-115.2 \pm 5.4$ & $-111.8(\mathrm{KS})$ \\
$N_{f}=5$ & $-10.07 \pm 1.99$ & -12.76 \\
& $-86.5 \pm 10.8$ & $-96.8(\mathrm{KS})$ \\
\hline
\end{tabular}

